\title{
Acessibilidade de um cadeirante em uma instituição pública do ensino superior: rotas e rotinas
}

\author{
Rita de Cássia Silveira Cambruzzi* \\ Maria da Piedade Resende da Costa** \\ Fátima Elisabeth Denari***
}

Resumo

A deficiência física como grupo é heterogêneo, mesmo dentro de uma "categoria", visto que abrange uma diversidade, pois cada um tem um mecanismo para se organizar nos espaços em que frequenta, mas todos participam dos mesmos desafios. Todavia, devido aos desafios encontrados pelas pessoas com deficiência física no estabelecimento de rotas e rotinas no espaço de uma universidade é que desenvolvemos a presente pesquisa. Optamos por pesquisar o acesso da pessoa com deficiência física no seu deslocamento externo da moradia estudantil até a sala de aula e, da sala de aula até a secretaria do departamento que freqüenta. A pesquisa cujo objetivo é a identificação e, análise das rotas quanto a sua acessibilidade, no campus de uma instituição pública de ensino superior. A pesquisa foi submetida ao Comitê de Ética que aprova a sua execução. Foram realizadas observações diretas, assim como registros através de fotografias e filmagens, que foram autorizadas pelo universitário. As informações coletadas foram analisadas qualitativamente conforme as normas da Associação Brasileiras de Normas Técnicas e o documento norteador do Conselho Regional de Engenharia e Arquitetura, do Rio Grande do Sul, intitulado: Roteiro de Vistoria. Na análise dos dados, constatamos de que há necessidades de alterações para propiciar à acessibilidade com segurança e comodidade, assim como, estabelecer mecanismos para que a biblioteca comunitária e o restaurante universitário façam parte da vivência como integrante da comunidade universitária e, também, no exercício de seus direitos constitucionais.

Palavras-chave: Acessibilidade; Educação especial; Deficiência física.

http://dx.doi.org/10.5902/1984686X4386

* Doutora pela Universidade Federal de São Carlos (UFSCar), e Psicóloga da Fundação Catarinense de Educação Especial. Sao Jose, Santa Catarina.

** Professora Doutora do Programa de Pós-Graduação em Educação Especial da Universidade Federal de São Carlos, São Carlos, São Paulo.

Professora Doutora do Programa de Pós-Graduação em Educação Especial da Universidade Federal de São Carlos, São Carlos, São Paulo. 


\title{
Accessibility of a cadeirante in a public institution of higher education: routes and routines
}

\begin{abstract}
The physical disability is group is heterogeneous, even within a "category", since that covers a diversity, because each has a mechanism to organize in spaces in which attends but all participate of the same challenges. However, due to the challenges encountered by persons with disabilities in establishing routes and routines within a University is that we have developed this research. We chose search access of physically disabled person in your external displacement of student housing to the classroom and the classroom until the Secretariat Department that frequents. Research whose goal is the identification and analysis of routes as its accessibility, on the campus of a public institution of higher education. The survey was submitted to the Ethics Committee that approves their implementation. Direct observations were performed, as well as records via photos and footage, which were authorized by the University. The information collected were analyzed qualitatively as the standards of the Brazilian Association of technical norms and the guiding document for the Regional Council of engineering and architecture, Rio Grande do Sul, titled: Roadmap survey. In data analysis, we see that there are needs for providing accessibility with safety and convenience, as well as establish mechanisms for the community library and the University restaurant part of living as part of the University community and also, in the exercise of their constitutional rights.
\end{abstract}

Keywords: Accessibility; Speciale education; Physical disabilities.

\section{Introdução}

No Brasil o Instituto Brasileiro de Geografia Estatística (IBGE 2000) revela o número de pessoas com deficiência é de 24,6 milhões e, nessa população, mais de nove (9) milhões possuem algum tipo de deficiência física (Associação de Assistência à Criança Deficiente - AACD, 2005).

Na clínica de Lesão Medular (AACD, 2005) realiza pesquisa onde $30,5 \%$ dos pacientes apresentaram lesão medular como conseqüência de acidente automobilístico. Nos acidentes de trânsito, as motos lideram com 
$43,6 \%$ e, 36,4\% representam o número de acidentes automobilísticos, $12,7 \%$ por atropelamento e, acidentes de bicicleta e caminhões apresentam uma incidência de $7,2 \%$. Além desses, há queda de altura (13\%) e mergulho em águas rasas $(6,8 \%)$ e, há um número expressivo de acidentes com armas de fogo (16 anos) causando danos sérios como a lesão medular.

O estudo aponta que a grande maioria $(62,7 \%)$, vive na grande São Paulo, no interior paulista estão $31,6 \%$ e, outros estados têm uma taxa de $5,7 \%$. Aponta que $83,5 \%$ são do sexo masculino com um aumento significativo de 10,2\%, além da última estatística.

Diante da pesquisa, podemos inferir que houve um aumento no número de pessoas com deficiência física no Brasil além de demonstrar a dimensão das dificuldades que essa população enfrenta no seu dia-a-dia.

A acessibilidade é vista, atualmente, como parte fundamental para o desenvolvimento das pessoas com deficiência com o objetivo de facilitar o acesso a diversos ambientes (MAZZONI, TORRES, OLIVEIRA, ELY e ALVES, 2001; DUARTE e COHEN, 2004; LOPES, DUTRA, MARTELETO, GONÇALVES, GERALDO e LINHARES, 2007; MARCOS, OKIMOTO, SCHEER e WIGINESCKI, 2007; BERNARDI e KOWALTOWISKI, 2009; GUERREIRO e MUNSTER, 2010).

Por ser a universidade um espaço democrático suscitou inquietações no que se refere ao acesso aos diferentes espaços pela pessoa com deficiência física (cadeirante), entre outras, visto que há documentos legais que garantem a acessibilidade e, exigem a sua execução para tornálos acessíveis.

Aportes legais existem: leis federais, municipais, decretos, resoluções, que estabelecem o direito da pessoa com deficiência e, os estados da federação devem adotar a Lei Federal 10.098/00, que estabelece as normas gerais e os critérios básicos: promover a acessibilidade às pessoas com deficiência ou com mobilidade reduzida.

\section{Acessibilidade}

Duarte e Cohen (2004) após pesquisa com alunos, funcionários e docentes à respeito das barreiras arquitetônicas, na Universidade Federal do Rio de Janeiro, verificou que os grupos apresentaram diferentes dificuldades de locomoção e/ou visão. Os dados demonstram que: "há evidências da necessidade de uma arquitetura verdadeiramente inclusiva em uma 
universidade concebida para todos" (2004, p.1). As autoras, mais adiante (2004, p.2), enfatiza que: "planejamento de seus espaços deve permitir livre acesso de todos os segmentos da sociedade a todos os setores e níveis de ensino e pesquisa".

Marcos et al (2007, p. 1) relatam que:

o portador de deficiência física motora é um dos indivíduos mais fortemente penalizados pela falta de acessibilidade do espaço urbano e edificado, pois sua mobilidade depende do uso de cadeira de rodas, e o ambiente construído ainda está muito pouco adaptado para garantir o seu direito de ir e vir.

Os autores observaram o grau de dificuldade para se locomover, indicando problemas de acessibilidade dentro e fora da moradia (MARCOS et al, 2007).

Moraes (2007, p. 10) aplica as normas técnicas ao espaço construído para conferir se o ambiente pode ser um facilitador [...] "garantindo o direito de igualdade sem nenhuma forma de discriminação entre as pessoas, ou um agente que muitas vezes dificulta ou impede a utilização do espaço construído por todas as pessoas".

Lopes et al (20077, p. 2): "A mobilidade com autonomia e segurança, constitui um direito universal e resulta das conquistas sociais e do conceito de cidadania". Os autores assinalam que: "O ambiente é de extrema importância no dia a dia de todos e a responsabilidade de ter uma relação de bem estar com as pessoas que o utilizam". E, mais adiante: "[...] estamos longe de poder favorecer a todos o direito de ir e vir. [...]", de acordo com Lopes et al $(2007$, p. 2)

Bernardi e Kowaltowski (2009, p. 1456) relatam que os resultados da pesquisa "demonstram que o processo de projeto deve ocorrer de forma participativa e que novas formas de comunicação são necessárias". Dessa forma, os novos profissionais terão atitudes sensíveis às diversidades tomando como referência o conceito do Desenho Universal quando dos projetos a serem planejados (BERNARDI E KOWALTOWSKI, 2009).

Destefani (2009, p. 2) desenvolveu uma pesquisa no Espaço Arquitetônico da Biblioteca na Universidade de Campinas com deficientes visuais com a "finalidade de aprimorar um instrumento de leitura tátil já 
existente". Segundo a autora a acessibilidade é vista como parte fundamental para o desenvolvimento das pessoas com deficiência.

Guerreiro \& Munster (2010, p. 6), citando Assunção e Araújo (2008), enfatizam que: " o uso diferenciado da cidade demonstra que o espaço se constrói e se reproduz de forma desigual e contraditória, o que leva à desigualdade social e espacial, [...]".

Segundo a ABNT (BRASIL, 2004), devemos garantir o espaço para o trânsito das cadeiras de rodas sem nos esquecermos do conforto, da segurança e boa visibilidade, que no caso específico são pessoas com deficiência física, estarem integrados ao ambiente.

É importante observar dada a heterogeneidade que apresenta as pessoas com deficiência física, há necessidade de utilizar dos serviços durante a vida da pessoa, áreas de apoio, porque a autonomia das pessoas desse grupo também é diversa.

Estes aspectos são importantes a serem observados na instituição pública, nos diferentes espaços, para que a pessoa com deficiência física possa acessar todos os ambientes e, para que suas rotas e rotinas possam ser previstas, sem que cause impacto na acessibilidade e na vida acadêmica.

Diante do exposto surgem as seguintes questões: Quais os cuidados de prevenção que a universidade está realizando para o livre acesso aos espaços para as pessoas com deficiência, mais especificamente com a pessoa com deficiência física? Os espaços na universidade estão de acordo com as especificações técnicas?

Com o objetivo de responder estas questões o presente estudo teve como objetivo de identificar e verificar o deslocamento nas rotas e rotinas estabelecidas por um universitário (cadeirante) e, propor acesso alternativo disponível para torná-lo acessível.

\section{Metodologia}

Participou um universitário, do sexo masculino, de 20 anos de idade, cadeirante que freqüenta uma Universidade pública no interior do estado de São Paulo. 
A escolha pelos instrumentos: a. NBR 9050 Acessibilidade a edificações, mobiliário, espaços e equipamentos urbanos deu-se por ser 0 norteador quando da acessibilidade; b. Roteiro de Vistoria (CREA/RS, 2004) por tratar-se de protocolo categorizado de acordo com as especificações da norma e ser um facilitador prático, ao irmos a campo e, c. a Entrevista como complemento de informações.

A coleta de dados foi executada em diferentes etapas: 1. Submissão ao Comitê de Ética; 2.Convite e esclarecimento dos objetivos e do termo de compromisso livre e esclarecido (TCLE), incluindo autorização, voluntária, para a divulgação da imagem; 3. a rota / rotina do alojamento $(A)$, via prédio $B$, ao prédio das aulas teóricas (prédio $C$ ) e do prédio $C$ ao prédio $E$, foi realizada pelo estudante; 4 . Entrevista com o objetivo adquirir informações e, durante o trajeto a respeito do que estava sendo observado e vivenciado pelo estudante; 5. Filmagem e Fotografias durante a execução da rota, para identificar e evidenciar alguns aspectos a serem considerados, quando da proposição de modificações estruturais para a acessibilidade prevista na NBR9050 (2004).

Nesse processo houve uma busca na NBR 9050 para esclarecimentos evitando assim, erros em relação ao entendimento das colocações e, a validação das respostas, com o auxílio de uma profissional da área, em função alguns conhecimentos específicos como, por exemplo, à declividade das rampas encontradas no percurso das rotas e rotinas.

Inicialmente foram pensadas cinco rotas, a saber: a. rota 1, alojamento (A) até o local em que frequenta as aulas teóricas e que denominaremos prédio $C$, via prédio $B$; $b$. rota 2 , deslocamento do prédio $C$ ao prédio $E$, via prédio $D$ e, c. rota 3 , retorno do prédio $E$ a moradia $(A)$; d. rota 4, Restaurante Universitário e, a rota 5, Biblioteca Universitária.

Convém ressaltar que as rotas 4 e 5 foram filmadas com o objetivo de identificar as impossibilidades para o universitário em cadeira de rodas e, propor alternativas para se fazer acessível. Entretanto, todas as rotas foram submetidas ao documento de vistoria editado pelo CREA/RS e, observado as convenções utilizadas para avaliação (S - Sim, N - Não e, NE - não existe), tomando-as como parâmetros.

As rotas foram estabelecidas pelo estudante, filmadas e fotografadas nas partes mais críticas e usadas como apoio na avaliação, para identificá-las e caracterizá-las. Ainda em relação às rotas, os trajetos foram identificados por letras de $\mathrm{A}$ (alojamento), os prédios $\mathrm{B}$ e $\mathrm{C}$ - onde acontecessem as aulas, 
que no caso do universitário é o prédio $C$. Os prédios assinalados com a letra D, E, F, G, H e I referem-se aos prédios, que fazem ou que podem fazer parte da rota/rotina.

A Rota 1, alojamento (prédio $A$ ), via prédio $B$, ao prédio $C$ caracterizou-se por cinco trajetos, conforme quadro 1,a saber:

\begin{tabular}{|lc|}
\hline Quadro 1. Saída da moradia (prédio A) ao prédio C, via \\
\hline \multicolumn{2}{|c|}{ ROTA 1 } \\
\hline Trajeto 1 & Saida de A, via rampa, à calçada lateral de B \\
\hline Trajeto 2 & Rampa externa de acesso ao prédio B \\
\hline Trajeto 3 & Rampa interna de B e saída em direção a C \\
\hline Trajeto 4 & Rampa externa em direção à calçada lateral C \\
\hline Trajeto 5 & Calçada lateral C, rampa externa até Prédio C \\
\hline
\end{tabular}

A Rota 2, deslocamento do prédio $C$ ao prédio $E$, via prédio $D$, sendo assinalados três trajetos (Quadro 2).

\begin{tabular}{|lc|}
\hline Quadro 2. Deslocamento do prédio $\mathrm{C}$ ao prédio $\mathrm{E}$ \\
\hline & ROTA 2 \\
\hline Trajeto 1 & Saída de $\mathrm{C}$, via rampa externa, à calçada lateral de $\mathrm{C}$ \\
\hline Trajeto 2 & Saída rampa(final) à calçada rebaixada, via prédio $\mathrm{D}$ \\
\hline Trajeto 3 & Calçada rebaixada( prédio $\mathrm{D}$ ), travessia ao prédio $\mathrm{E}$ \\
\hline
\end{tabular}

Finalmente a rota 3 que indica o retorno do prédio $E$ ao prédio $A$. $A$ rota estabelecida pelo universitário foram desmembrados em quatro trajetos, de acordo o Quadro 3.

\begin{tabular}{|lc|}
\hline Quadro 3. Deslocamento do prédio E a moradia(A),via Quadras \\
\hline & ROTA 3 \\
\hline Trajeto 1 & Saída de E, travessia à faixa de pedestre, via asfalto \\
\hline Trajeto 2 & Faixa de pedestre à calçada rebaixada do prédio D \\
\hline Trajeto 3 & Calçada rebaixada(D) ao estacionamento, via asfalto \\
\hline Trajeto 4 & Calçada rebaixada do estacionamento à A, via Quadras \\
\hline
\end{tabular}


Em função das observações e das filmagens das rotas serão propostas rotas alternativas para o universitário e, as rotinas que no momento atual são as mesmas. Entretanto essas rotinas poderão ser expandidas se houver soluções viáveis.

\section{Resultados e discussão}

Na rota1, foram observados e registrados: as rampas, e as calçadas, como parte da sua vivência diária. Podemos verificar, conforme tabela 1, que a declividade varia de acordo com condição de localização. A rampa que dá acesso ao prédio $\mathrm{C}$, situada em frente à faixa de pedestre, tem como declividade $3,5 \%$, que é a menor entre elas, a do alojamento (prédio A) é $5,5 \%$ e, a que conduz ao prédio C, registra uma declividade de $7 \%$.

Tabela 1.Rampa \& Declividade ( \% )

\begin{tabular}{c|c|c|c|c}
\hline Prédio A & Acesso a B & Interno B & Saída B \& C & Prédio C \\
\hline & & & & \\
\hline 5,5 & 12,5 & 11 & Inicial & 7 \\
\hline & & & 14,5 & \\
\hline & & & Intermediária & \\
\hline & & & 14,5 & \\
\hline & & & Final & \\
\hline & & & 14,5 & \\
\hline
\end{tabular}

Os registros estão em desacordo com os parâmetros da NBR9050 (2004, p.42, tabela5) que variam de 5,00 a 8,33\%, com exceção da localizada antes da faixa de pedestre, que dá acesso ao prédio 2 . As rampas em que o universitário faz uso ao se deslocar até o prédio $\mathrm{C}$, apresentam uma variação de $14,5 \%$, na rampa inicial e intermediária, e $15,5 \%$ no trecho final da rampa em direção a calçada lateral (prédio $\mathrm{C}$ ). Já no prédio $\mathrm{B}$, a rampa externa apresenta uma declividade de $12,7 \%$ e, a rampa interna é $11 \%$. Portanto, diante dos dados, só a rampa do alojamento (prédio A), rampa de acesso ao prédio $\mathrm{C}$ e, a do prédio $\mathrm{C}$, propriamente dita, está de acordo com o padrão mínimo e, as demais em desacordo.

Segundo as normas, há um dimensionamento de rampa para quando foram esgotadas as possibilidades de solução, como a de reformas, e os critérios estabelecidos podem variar de $8,33 \%$ até $12,5 \%$, conforme a referência normativa: 6.5.1.3. Entretanto, o trecho que estabelece a ligação entre a parte baixa (prédio B), com a Rua onde frequenta as aulas (prédio C) 
a declividade é mais acentuada; significa dizer, que o universitário desprende muita energia para transpô-las. ${ }^{1}$

A tabela 2 demonstra que a largura das rampas é variável tomando como parâmetros normativos (NBR 9050, 2004) de que a largura mínima estabelecida é de $1,20 \mathrm{~m}$. Observamos que a largura da rampa (prédio A) está fora dos parâmetros estabelecidos, mas apesar desse aspecto, o universitário a transpõe tranquilamente, mas não comporta uma pessoa ao lado, por ter como medida $1,14 \mathrm{~m}$.

Tabela 2. Rampa \& Largura ( $m$ )

\begin{tabular}{c|c|c|c|c}
\hline Prédio A & Acesso a B & Interno B & Saída B \& C & Prédio C \\
\hline & & & & \\
\hline 1,14 & Inicial & 1,2 & Inicial & Conforme \\
\hline & 1,40 & & 1,37 & NBR 9050 \\
\hline & Final & & Intermediária & \\
\hline & 2,59 & & 1,38 & \\
\hline & & & Final & \\
\hline & & & 1,38 & \\
\hline
\end{tabular}

Na rota 1, em relação ao piso da rampa, verificamos que: a da moradia, a de acesso a faixa de pedestre e a do prédio $C$, em sua extensão, apresentam um piso antiderrapante sob todas as condições e, satisfatório se considerarmos que não há "escavados", rachaduras. Entretanto, a rampa de ingresso ao prédio $\mathrm{B}$ (calçada lateral) está com rachaduras, depressões e consertos inadequados, o que provoca a trepidação da cadeira.

Já a rampa interna do prédio $\mathrm{B}$ (salas de aulas), apresenta 'rachaduras' e, as que promovem a ligação do prédio $B$ à via lateral do prédio C apresentam piso "escavado" e desmoronamento da lateral, observável. Portanto, as rampas estão mal conservadas.

As laterais das rampas são protegidas por paredes e corrimãos $(A)$, no prédio $B$ por paredes e a do prédio $C$, por corrimãos.

Convêm esclarecer, que há exceção no trecho desde a saída do prédio $B$ nas três rampas externas, em que há corrimão $(0,90 m)$ em um só lado, o pode provocar acidente, pois nas laterais da rampa a altura varia de $0,5 \mathrm{~m}$ (parte baixa) e, alcança $0,41 \mathrm{~m}$ (alta). 
Foram observados patamares entre a rampa inicial e a intermediária e da intermediária ao final da rampa e, que parecem estar em concordância com a norma, colocado em direção ao movimento.

Em relação às rampas, segundo a NBR9050, as mesmas devem estar sinalizadas no início e no final e, a sinalização de alerta e direcional foram observadas somente na rampa do prédio $\mathrm{C}$ e, as demais não existem.

As três rotas analisadas indicam que a maioria das calçadas está em concordância com as referências normativas (6.1 e 6.10). As calçadas variam de largura nos trajetos das rotas e, são consideradas adequadas, porque atendem a medida mínima estabelecida; os pisos na grande maioria são antiderrapantes. Todavia, em alguns trechos (rota1) há comprometimentos em função de buracos, fissuras, depressões, espaços sem a camada de piso e com vegetação.

A calçada lateral que dá acesso à rampa do prédio $C$ é exceção. Nesse trajeto, especialmente, a largura é de 1,20m, conforme a norma, se não houvesse vegetação lateral (arbustos) em toda a sua extensão e, que necessitaria 1,50m de largura. Apesar de ser contínuo o deslocamento, essa vegetação, provoca no universitário uma postura de afastamento para evitar acidentes e, resulta em espaço reduzido para uma pessoa acompanhá-lo, com segurança.

No trajeto, que corresponde a saída da rampa do prédio $C$ até o acesso para o prédio $\mathrm{D}$ (rota 2 ), há a necessidade de dividi-lo: a primeira $\left(1^{\mathrm{a}}\right)$, logo após a saída, a calçada é larga, mas o estado de conservação da superfície da calçada segundo Keppe Júnior (2007, p. 67), apresenta "condições precárias" e, as caracteriza como "alguns buracos ou irregularidades". O universitário comenta que "a largura é boa, mas os buracos é que são ruins" (sic) ao deslocar-se e, necessita desviar-se de um lado para outro para encontrar um trecho sem buracos e depressões.

A continuidade $\left(2^{\mathrm{a}}\right)$ se refere à calçada anterior ao piso intertravado (prédio D), o estado de conservação é caracterizado por Keppe Júnior (2007, p.67) como "condições ruins", pois "encontramos irregularidades". Observamos irregularidades significativas como pedaços das calçadas sem a cobertura, depressões e com vegetação.

Na calçada lateral do prédio $D$ é livre de obstáculos, o piso é firme e oferece boas condições para o deslocamento com a cadeira de rodas, pois não há depressões durante a passagem. A largura efetiva da calçada é de 
$1,50 \mathrm{~m}$, contado com o meio-fio, o que possibilita o deslocamento de uma pessoa em cadeira de rodas e, um acompanhante.

As calçadas em direção ao prédio $E$ são largas, com piso antiderrapante, mas no trajeto há tampa de caixa levantada e afastada. Essa caixa está próxima ao prédio $\mathrm{F}$ havendo necessidade de desviar-se e, andar junto à parede.

O retorno a moradia $(A)$, que corresponde a rota 3 , iniciou em área contígua a calçada de acesso a secretaria do curso (prédio $\mathrm{E}$ ), que apresentava boas condições quanto ao piso, à movimentação da cadeira de rodas, mas ao alcançar a calçada lateral do prédio $\mathrm{F}$ os problemas surgiram.

No referindo trajeto verificamos que: há piso tátil direcional quebrado, grelhas danificadas, desníveis, encaixes mal feitos nas juntas de dilatação provocando trepidação e, por não atender as normativas para a sua colocação. Segundo a norma NBR9050 (2004, p.40) "as grelhas e juntas de dilatação devem estar preferencialmente fora do fluxo principal de circulação" e, nas rotas acessíveis se 'instalados transversalmente' e, os vãos devem ter 'dimensão máxima de 15mm'.

Percebemos que no trajeto havia 'consertos mal feitos' para substituir a sinalização, tátil de alerta e direcional junto à calçada. meio fio 'desmoronado' e má conservação nas laterais da calçada.

A rota alternativa para o retorno do prédio $\mathrm{E}$ para a moradia (prédio A) iniciava o trajeto do prédio $F$ (lateral) até a calçada rebaixada que dá acesso a calçada lateral do prédio $\mathrm{G}$ e, seguir até a bifurcação existente no acesso ao prédio $\mathrm{H}$. Entretanto, devem ser feitas melhorias nos trechos devido à precariedade em alguns trechos: calçada rebaixada e lateral danificada (prédio $\mathrm{G}$ ); a calçada em bifurcação (prédio $\mathrm{H}$ ) apresenta desníveis, caixa com tampa levantada /solta e piso danificado.

Na lateral (prédio H) há um acesso onde é possível fazer a travessia em cadeira de rodas, pois a largura da passagem é de $1,20 \mathrm{~m}$ e, é possível fazer manobras. A largura da calçada é $1,20 \mathrm{~m}$ (início) e fica reduzida porque há vegetação e tampa de caixa deslocada, sobre a mesma. No final da calçada (prédio $\mathrm{H}$ ), no sentido da via, deve haver sinalização indicativa e, uma faixa de pedestre para a calçada rebaixada que está no lado oposto (prédio D) para a travessia segura. 
As ações, realizadas pela a universidade, facilitariam o trajeto e, provavelmente, pouparia um desprendimento de energia maior do que necessário; ou então, seguir pela calçada lateral (prédio C), se deslocar pela rampa, frente à faixa de pedestre. Retornar ao destino final pelas rampas do prédio I e, prosseguir pela calçada até o entroncamento com a lateral das Quadras Esportivas para, se deslocar até a moradia.

Em relação à rota 4 - o Restaurante Universitário (RU), nas condições atuais é impossível o acesso, pois não há rota acessível.

Como resultado do instrumento Vistoria (CREA/RS, 2007) que analisa restaurantes, refeitórios, bares e similares verificamos, que: a. não existem mesas acessíveis, para a pessoa em cadeira de roda conforme a exigência da norma, que é de pelo menos $5 \%$ do total das mesas; b. faixa livre de circulação e área de manobra para acesso as mesas; c. balcões para refeições acessíveis e localizadas junto à rota acessível. Vale ressaltar, que alguns aspectos que necessitavam aferições não foram avaliados.

A rota 5 , que corresponde a da Biblioteca Comunitária, foi a outra rota registrada e, que atualmente, não tem acesso. Essa rota é eminentemente realizada através de escadas em diferentes alturas e, após vencê-las há um caminho a ser percorrido pelos universitários. Há que enfatizar, de que há sinalização de alerta na saída da calçada e piso tátil direcional durante todo o trajeto, até chegar à via Biblioteca Comunitária. Entretanto, em um trecho antes de chegar à avenida, há uma árvore, que pode ser perigosa para quem é deficiente visual ou com baixa visão; a mesma está na rota do usuário e, a sua altura parece estar abaixo de $2,00 \mathrm{~m}$, pois houve a necessidade de desviar de seus ramos, como aconteceu no deslocamento.

Outra observação relevante é, que em um trecho da calçada em direção a biblioteca comunitária, não é adequada a todas as pessoas, conforme o desenho universal. Para uma pessoa em cadeira de roda é impraticável e, acrescentado ao que já foi dito, há ainda automóveis que ficam sobre a faixa de pedestre, que dificultam e obstruem o acesso para as pessoas.

A rampa de acesso a Biblioteca Comunitária também deparamos com bicicletas, acorrentadas no corrimão o que pode causar acidente e, por impedir a continuidade do trajeto para quem precisa utilizá-lo. A rampa é larga, mas não nos foi possível discernir se a declividade está ou não de acordo com os parâmetros normativos, pois não utilizamos de instrumento para aferi-la. 
Na Biblioteca Comunitária, o instrumento não foi aplicado, mas pela vivência no ambiente, acredito que não está conforme a normas técnicas (NBR 9050, 2004) ou, pelo menos adaptadas em alguns aspectos que possibilitem o universitário com deficiências, seja cadeirante, visual ou com baixa visão, por exemplo, ter acesso à consulta de livros, que é imprescindível na formação acadêmica.

Sabe-se, que hoje há mecanismos que viabilize a rota 4 como alternativa: rota com ônibus adaptado, havendo uma parada no espaço lateral que dá acesso a cozinha do RU(Rua do Lago). Nesse local, encontramos uma calçada que dá acesso a porta do R.U, entretanto, as condições de conservação são críticas. Há a necessidade de reestruturação física de toda a sua extensão, verificação da largura da calçada, conforme as normas estabelecidas, consertos para a locomoção segura.

Quanto à alternativa para o acesso a biblioteca seria a garantia de um ônibus coletivo adaptado, de forma adequada, para que o universitário possa acessar como todos os outros da comunidade acadêmica, participando como integrante e, tendo o direito de aprimorar em seus conhecimentos. Outros virão assim que a universidade estiver acessível para receber os deficientes físicos, sejam cadeirantes ou com mobilidade reduzida, foco da nossa pesquisa.

\section{Conclusão e recomendações}

Diante dos dados, podemos verificar que as considerações apontadas por pesquisadores são pertinentes quanto à acessibilidade: 1 . há necessidade de uma arquitetura inclusiva; 2 . que os espaços públicos ofereçam acessibilidade aos usuários em cadeira de rodas; 3 . que mudanças atitudinais ocorram para atender as diversidades, resultando em autonomia e segurança, já que os deficientes físicos são os mais penalizados no universo das pessoas com deficiência.

A análise nos fornece informações e nos permite favorecer a pessoa em cadeira de rodas com ações corretivas e preventivas. Essas ações devem eliminar as contradições entre o real e o previsto pelas normas estabelecidas, respaldado em dispositivos legais, para garantir o direito constitucional de ir e vir.

Verificamos que há necessidade de intervenções planejadas e execução das mesmas para a manutenção (calçadas e calçadas rebaixadas, 
rampas) através de serviço cuidadoso, evitando ressaltos que impedem o deslocamento sem trepidação na transposição dos obstáculos.

Recomenda-se de que seja realizado um estudo em que viabilize o acesso viável e seguro ao estudante em cadeira de rodas, rotas (4 e 5) que são inviáveis diante das barreiras que apresentam; garantido rotas alternativas para acesso aos serviços instituídos; e, o espaço universitário em condições de igualdade com os não deficientes.

Acreditamos que a universidade, enquanto espaço público e democrático formalizaria o que está previsto nos princípios da escola inclusiva, nos documentos legais, que garantam a acessibilidade no diferentes níveis.

\section{Referências}

BRASIL. Associação Brasileira de Normas Técnicas. NBR9050: Acessibilidade a edificações, mobiliário, espaços e equipamentos urbanos. Rio de Janeiro: ABNT, 2004. 98p

BRASIL. Associação de Assistência à Criança Deficiente - AACD. Clínica de Lesão Medular. São Paulo, 2005.

BRASIL. Instituto Brasileiro de Geografia e Estatística. Censo demográfico 2000: população residente, por tipo de deficiência. Brasília, 2001.

BRASIL. Presidência da República. Casa Civil. Subchefia para Assuntos Jurídicos. Lei $\mathbf{n} . \mathbf{1 0 . 0 9 8}$ de 19 de dezembro de 2000. Disponível em:< http:// www.planalto.gov. br/ccivil_03/ leis/L10098.htm>. Acesso em: 22 mai. 2010.

BERNARDI, N.; KOWALTOWSKI, D. C. C. K. A aplicação do conceito do desenho universal no ensino da arquitetura: Considerações sobre o uso de instrumento de leitura de projeto. In: ENCONTRO NACIONAL e VIENCONTRO LATINO AMERICANO DE CONFORTO NO AMBIENTE CONSTRUÍDO, 2009, Natal. Anais... Natal: ENCAC e ELAC, 2009. p.1456 - 1465.

DESTEFANI, A. Verificação da usabilidade de instrumento de leitura tátil e sonoro representativo do espaço arquitetônico da biblioteca central Cesar Lattes da Unicamp. Relatório Final. Universidade Estadual de Campinas/Departamento de Arquitetura e Construção. Campinas, 2009, 19p. 
DUARTE, R. S.; COHEN, R. Acessibilidade aos espaços do ensino e pesquisa: desenho universal na UFRJ - possível ou utópico? In: NUTAU 2004: DEMANDAS SOCIAIS, INOVAÇÕES TECNOLÓGICAS E A CIDADE, 2004, São Paulo. Anais... São Paulo: NUTAU 2004: demandas sociais, inovações tecnológicas e a cidade, 2004.

GUERREIRO, E. M. B. R. \& MUNSTER, M. A. van. Acessibilidade física de alunos com deficiência visual no ensino superior. Programa de PósGraduação em Educação Especial /Universidade Federal de São Carlos. São Carlos, 2010.

KEPPA JUNIOR, C. L. G. Formulação de um indicador de acessibilidade das calçadas e travessias. Dissertação (Mestrado em Engenharia Civil). Universidade Federal de São Carlos. São Carlos, 2007.152f.

LOPES, K. T. et al. Acessibilidade de pessoas deficientes em escolas Públicas. Disponível em: <http://www.wgate.com.br/ conteudo/ medicinaesaude/fisioterapia/variedades/ acessibilidade_ gleisson.htm>. Acesso em: 22 mai.2010.

MAZZONI, A. A. et al. Aspectos que Interferem na Construção da Acessibilidade em Bibliotecas Universitárias. Ciência da Informação. Brasília - DF - Brasil: v.30, n.2, p.29 - 34, 2001. Disponível em: <http://www. scielo.br/pdf/ci/v30n2/6209.pdf>. Acesso em: 22 mai. 2010.

MARCOS, M. et al. Avaliação e análise de acessibilidade de um deficiente físico motor, através do software Catia, em habitações de interesse social. UFRP, 2007. Disponível em: <www.cesec.ufpr.br/workshop2007/ Artigo-25.pdf>. Acesso em: 22 mai. 2010

MORAES, M. C de. Acessibilidade no Brasil: análise da NBR 9050. Dissertação (Mestrado em Arquitetura e Urbanismo). Universidade Federal de Santa Catarina, Florianópolis, 2007.173f.

RIO GRANDE DO SUL. Conselho Regional Engenharia Arquitetura e Agronomia do Rio Grande do Sul. Roteiro de Vistoria: Acessibilidade a edificações, mobiliário, espaços e equipamentos urbanos. Revisado. Coordenação: Orildes Tres. Porto Alegre: CREA/RS 2007. 23p. 
Rita de Cássia Silveira Cambruzzi - Maria da Piedade Resende da Costa - Fátima Elisabeth Denari

Notas

${ }^{1}$ Não existem rampas nas rotas 2 e 3

\section{Correspondência}

Rita de Cássia Silveira Cambruzzi - Fundação Catarinense de Educação Especial, Centro de Atendimento a Deficiência Sensorial Ceads. Rua Paulino Pedro Hermes, 2785, Nossa Senhora do Rosário, CEP: 88108-500 - Sao Jose, Santa Catarina - Brasil.

E-mail: ritacambruzzi@yahoo.com.br

Recebido em 29 de janeiro de 2012

Aprovado em 01 de março de 2013 